\title{
Phase-manipulation-induced Majorana Mode and Braiding Realization in Iron-based Superconductor $\mathrm{Fe}(\mathrm{Te}, \mathrm{Se})$
}

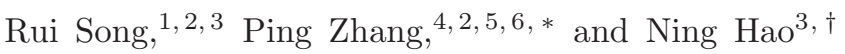 \\ ${ }^{1}$ HEDPS, Center for Applied Physics and Technology and School of Physics, Peking University, Beijing 100871, China \\ ${ }^{2}$ HEDPS, Center for Applied Physics and Technology and School of Engineering, Peking University, Beijing 100871, China \\ ${ }^{3}$ Anhui Key Laboratory of Condensed Matter Physics at Extreme Conditions, \\ High Magnetic Field Laboratory, HFIPS, Anhui, Chinese Academy of Sciences, \\ and University of Science and Technology of China, Hefei, China \\ ${ }^{4}$ School of Physics and Physical Engineering, Qufu Normal University, Qufu 273165, China \\ ${ }^{5}$ Institute of Applied Physics and Computational Mathematics, Beijing 100088, China \\ ${ }^{6}$ Beijing Computational Science Research Center, Beijing 100084, China
}

\begin{abstract}
Recent experiment reported the evidence of dispersing one-dimensional Majorana mode trapped by the crystalline domain walls in $\mathrm{FeSe}_{0.45} \mathrm{Te}_{0.55}$. Here, we perform the first-principles calculations to show that iron atoms in the domain wall spontaneously form the ferromagnetic order in line with orientation of the wall. The ferromagnetism can impose a $\pi$ phase difference between the domainwall-separated surface superconducting regimes under the appropriate width and magnetization of the wall. Accordingly, the topological surface superconducting state of $\mathrm{FeSe}_{0.45} \mathrm{Te}_{0.55}$ can give rise to one-dimensional Majorana modes trapped by the wall. More interestingly, we further propose a surface junction in the form of $\mathrm{FeSe}_{0.45} \mathrm{Te}_{0.55} /$ ferromagnet/ $\mathrm{FeSe}_{0.45} \mathrm{Te}_{0.55}$, which can be adopted to create and fuse the Majorana zero modes through controlling the width or magnetization of the interior ferromagnetic barrier. The braiding and readout of Majorana zero modes can be realized by the designed device. Such surface junction has the potential application in the superconducting topological quantum computation.
\end{abstract}

Majorana zero modes (MZMs) have attracted intense attention in condensed matter physics for the potential application in topological quantum computation [1 $[6]$. The paradigm for pursuing them lies in inducing Cooper pairs to the spin-momentum-locked bands through superconducting proximity effect in the artificial physical systems. The candidate platforms mainly includes the topological insulator, the Rashba-spin-orbit-coupled semiconductor and the ferromagnetic atomic chains forming the heterostructures with the conventional s-wave superconductors [7 11]. However, the weakness of the proximityeffect-induced superconductivity requires the ultra-low temperature and limits the potential applications.

Recently, the electronic bands with nontrivial topology have been theoretically predicted and experimentally verified in some iron-based superconductors 12 17. The coexistence of topology and high-temperature superconductivity make the iron-based superconductors as the unique platform to realize the high-temperature topological superconductors 15, 18 26. In general, some defects can trap the MZMs in topological superconductors. Assisted by angle resolved photoelectron spectroscope (ARPES), the scanning tunnel microscope/spectrum (STM/S) has observed the zero-energy conductance anomalies located on some point defect such as iron impurity and superconducting vortex in $\mathrm{Fe}(\mathrm{Se}, \mathrm{Te}), \mathrm{Li}_{1-x} \mathrm{Fe}_{x} \mathrm{HOFeSe}$, $\mathrm{CaKFe}_{4} \mathrm{As}_{4}$, and $\mathrm{Fe}(\mathrm{Se}, \mathrm{Te}) / \mathrm{STO}$, which strongly indicate the emergence of MZMs 27 35]. Unlike the point-like MZMs, more recently, an unexpected one-dimensional (1D) dispersive Majorana mode trapped by the crystalline domain walls (DWs) in $\mathrm{FeSe}_{0.45} \mathrm{Te}_{0.55}$ have been experimentally reported [36]. It is found that the DWs and 1D Majorana mode show some interesting features. For instance, the lattices form the bulge structure approaching the DWs. The orientation of the DW has a deflection of about a 45 degree angle against the direction of lattice shift. The differential conductance $d I / d V$ spectra show some subtle differences at the different positions of DWs. However, the physical origin and the features of the 1D Majorana mode have not been comprehensively understood.

In this work, we perform the first-principles calculations to investigate the properties of the DWs in $\mathrm{FeSe}_{0.45} \mathrm{Te}_{0.55}$. The numerical results can capture both the bulge structure and the specific orientation of the DWs. Interestingly, we find that the iron atoms in the DWs spontaneously form the ferromagnetic order with the magnetization direction along the DWs. Accordingly, we show that the ferromagnetism can manipulate the surface superconducting phase difference between the two sides of the DWs, which is analogous to the physical picture of Fulde-Ferrell-Larkin-Ovchinnikov (FFLO) superconducting state [37, 38]. The appropriate width and magnetization of the DWs can give $\pi$ phase difference and induce 1D Majorana mode. The fluctuations of the width and magnetization of the DWs make the phase difference to slightly deviate from $\pi$, which results in the subtle differences between the position-dependent $d I / d V$ spectra. More meaningfully, we further propose a surface junction in the form of $\mathrm{FeSe}_{0.45} \mathrm{Te}_{0.55} /$ ferromagnet $/ \mathrm{FeSe}_{0.45} \mathrm{Te}_{0.55}$ $(\mathrm{S} / \mathrm{F} / \mathrm{S})$. Tuning the width or magnetization in the different regimes of the ferromagnet, the surface junction can be adopted to create and fuse the MZMs located on the boundary between different regimes. The braiding 
and readout of MZMs can be realized by the designed device based on such junction.

As the STM experiment shows, the DWs are a line of dislocation-like defects, which separate the lattices into left and right parts with a relative half-unit-cell shift 36]. The thickness of DWs is one or several unit cells of $\mathrm{Fe}(\mathrm{Te}, \mathrm{Se})$ from experimental observation [39]. Therefore, we construct a $14 \times 1 \times 1$ supercell of $\mathrm{Fe}(\mathrm{Te}, \mathrm{Se})$, and the left and right seven unit cells are connected by a horizontal mirror reflection about iron plane. The calculation details are present in Ref. [40] . The stable structure is displayed in Fig. 1(a)-(c), with a obvious bulge structure deformation. The simulated STM image in Fig. 11(d) reveals that the DWs are much lighter than other regions in accord with the experimental observations 40]. Furthermore, the deflection of about a 45 degree angle of the orientation of the DW against the direction of half-unitcell shift is also captured by the simulation in comparison with image in Fig. 3(d) of Ref. 36]. The bulge structure deformation is due to the unsymmetric $\mathrm{Fe}-\mathrm{Te}(\mathrm{Se})$ chemical bonds in the DWs, which break the force balance along $\mathbf{z}$ direction. Another impact from the unsymmetric bonds is to enhance the localization of irons atoms in the DWs, which have a tendency to generate the magnetic moments. To determine the possible magnetic order of the DWs, for convenience, we still assume the $\mathrm{Fe}(\mathrm{Te}, \mathrm{Se})$ at the two sides of DWs possess the antiferromagnetic (AFM) order in spite of only strong AFM fluctuation existing in $\mathrm{Fe}(\mathrm{Se}, \mathrm{Te})$. This is the general strategy adopted by the calculations to determine the magnetism of ironbased superconductors 51 53]. It is well accepted that the superconducting state in $\mathrm{Fe}(\mathrm{Te}, \mathrm{Se})$ has a close relationship with the collinear AFM order, which we consider as shown in Fig. 1 (a) and (b). For the iron atoms in the DWs, we consider the AFM and FM pattern in Fig. 1 (a) and (b), respectively. The ground-state magnetic pattern is self-consistently calculated and determined. As shown in Fig. 1 (e), the magnetic pattern in Fig. 1 (b) has lower energy than that in Fig. 1 (a). The Hubbard $\mathrm{U}$ can further enlarge the energy difference. The calculated results are not unexpected, because the position switchings of the anions do not dramatically modulate the amplitudes of the nearest and next nearest neighbor exchange couplings. According to the $J_{1}-J_{2}$ model 53 [55], the magnetic pattern shown in Fig. 1 (b) is reasonable. Note that we also calculate the lattice pattern with domain walls involving two lines of irons. The same ferromagnetic order is also obtained [40]. Note that the local ferromagnetism in $\mathrm{Fe}(\mathrm{Te}, \mathrm{Se})$ was verified by recent experiments [56, 57].

Below superconducting transition temperature $T_{c}$, the superconductivity arises through suppressing the collinear AFM order or spin fluctuation in normal state, while the FM order in the DWs is preserved due to the absence of superconductivity. Then, the system can be simplified as a $\mathrm{S} / \mathrm{F} / \mathrm{S}$ junction which only involving the

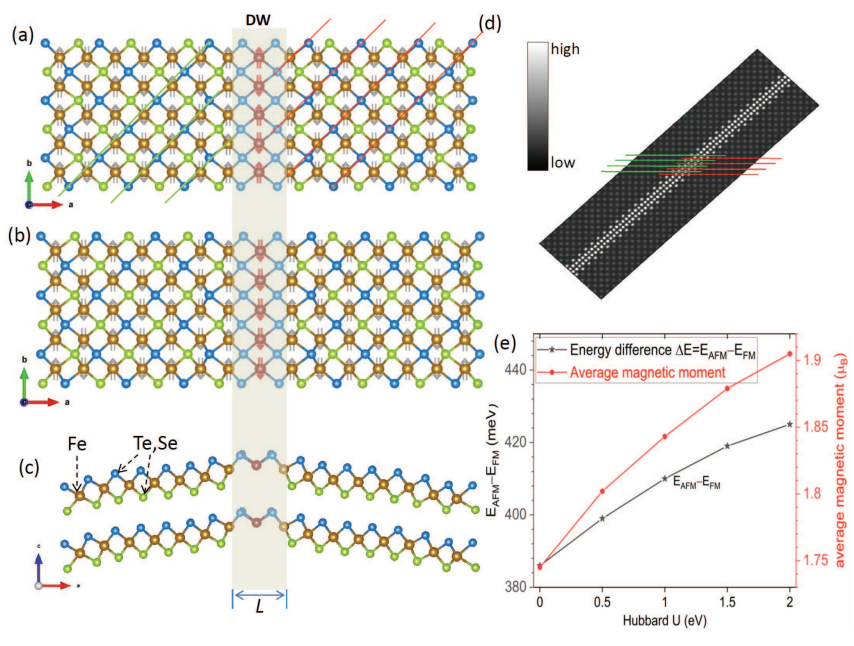

FIG. 1: (a)-(c) Top and side view of $\mathrm{Fe}(\mathrm{Te}$,Se) with $14 \times 1 \times 1$ super cell with DW labeled by the shadowed region. In both (a) and (b), the two sides of the DW have the collinear AFM order, but the iron atoms have the AFM and FM order in the DW in (a) and (b), respectively. (d) The simulated STM pattern. (e) The magnetic moment and the energy difference between the magnetic patterns in (a) and (b), i.e., $E_{A F M}-$ $E_{F M}$.

topological surface states, as shown in Fig. 2(a). The ferromagnetism of the DWs could generate an exchange field, i.e., $h_{y}=\left(n\left\langle S_{i}^{y}\right\rangle / \mu_{B}\right) \int J(\mathbf{r}) d^{3} r$, with $n, S_{i}^{y}, \mu_{B}$, and $J(\mathbf{r})$ labeling the concentration of localized moments, the average value of the localized spins, Bohr magneton and ferromagnetic exchange integral, respectively. Hereafter, we set $\mu_{B}=1$ for convenience. It has been shown that the F layer can generally modulate a superconducting phase difference between the two S layers. Here, we give a simple picture to address this point. Consider the normal Fermi surface of both $\mathrm{S}$ layer described by the topological surface Hamiltonian $H_{\text {surf }}=v_{F}(\sigma \times \mathbf{k}) \cdot \hat{z}$, the Fermi surface of the right $\mathrm{S}$ layer is shifted $\mathbf{Q}=h_{y} / v_{F} \hat{\mathbf{k}}_{x}$ by the effective Zeeman term $g \sigma_{y} h_{y} / 2$ in reference to the one of the left $\mathrm{S}$ layer. From the first-principles calculations, we estimate the effective Zeeman energy $h_{y} \sim 55 \mathrm{meV}[40]$. The Fermi velocity $v_{F} \sim 216 \mathrm{meV} \AA$ and $k_{F} \sim 0.03 \AA^{-1}[58]$. Thus $Q \sim 0.25 \AA^{-1}$, and $Q \gg k_{F}$. The left and right Fermi surfaces are fully separated, as shown in Fig. 2(b). The superconducting order parameter $\Delta(\mathbf{Q})$ is proportional to $\left\langle\hat{c}_{\mathbf{k} \uparrow} \hat{c}_{2} \mathbf{Q}-\mathbf{k} \downarrow\right\rangle$, which becomes spatially oscillating after Fourier transform into the real space. Namely, $\Delta(\mathbf{R})=\Delta_{0} e^{i 2 \mathbf{Q} \cdot \mathbf{R}}$ with $\Delta_{0}$, $\mathbf{R}$ labeling the induced superconducting order parameter of the topological surface band and the coordinate of center of mass of Cooper pair, respectively. This is equivalent to stating that a Cooper pair propagates across a Zeeman field and acquire a finite momentum and a phase oscillation in the real space, which is similar to the FFLO state [37, 38]. The phase shift is related to the width $L$ 

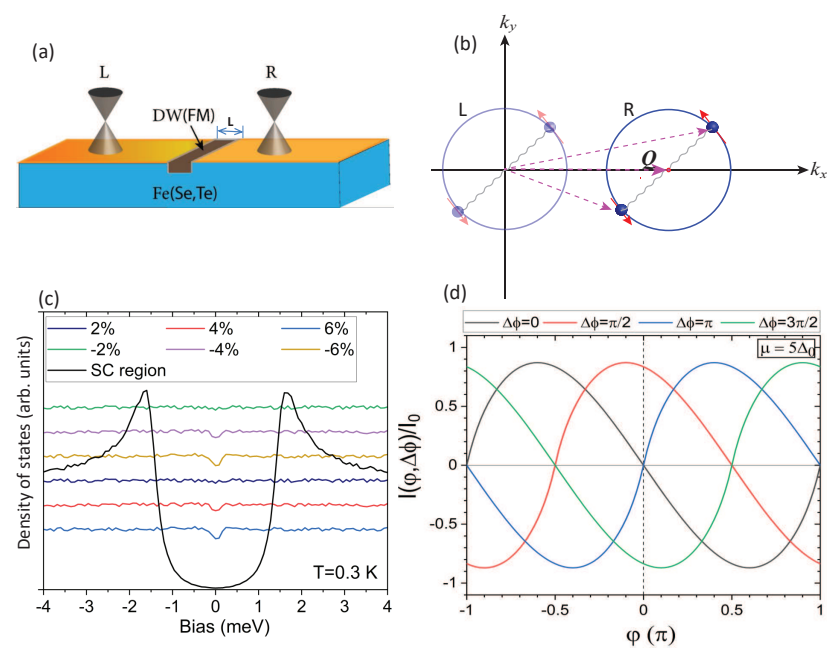

FIG. 2: (a) Schematic plotting for the surface S/F/S junction. The left $(\mathrm{L})$ and right $(\mathrm{R})$ gray Dirac cones denote the topological surface bands of $\mathrm{Fe}(\mathrm{Te}, \mathrm{Se})$, and the middle labels the ferromagnetic DW. (b) The Fermi surface of the R Dirac cone shifts momentum $Q$ in the reference of $\mathrm{L}$ Dirac cone. The pairing is indicated by the wave lines. (c) The calculated density of states (DOS) for the different $\Delta \phi$ fluctuations $[-6 \%$, $6 \%$ ] about $\pi$. The U-shape DOS is for the uniform superconducting state. The temperature is $0.3 \mathrm{~K}$. (d) The oscillation of tunneling current $I(\varphi, \Delta \phi)$ about $\varphi$ for different $\Delta \phi$. Note that the current is not zero for $\varphi=0$ and $\Delta \phi \neq 0, \pi$, which is different from the conventional junction.

and intensity of the effective Zeeman field $h_{y}$ of DWs and can be expressed as $\Delta \phi=2 h_{y} L / v_{F}$. When the system is in equilibrium state, no random phase difference occurs between the left and right superconducting regions, however, the system becomes a surface $\Delta \phi$-junction due to the phase-shift effect. According to Fu and Kane's theorem [7], a 1D Majorana mode will arise in the DWs when $\Delta \phi=\pi$ in the limit of $L \rightarrow 0$.

To verify the argument, we consider the generic surface S/F/S junction with arbitrary width $L$ and effective Zeeman field $h_{y}$ of DWs, and solve the Dirac-Bogoliubov-de Gennes (BdG) equation,

$$
H_{B d G} \Psi=E \Psi
$$

with

$$
H_{B d G}=\left(\begin{array}{cc}
H_{0}(k) & i \sigma_{y} \Delta(r) \\
-i \sigma_{y} \Delta^{*}(r) & -H_{0}^{*}(-k)
\end{array}\right) .
$$

Here, $H_{0}(k)=v_{F}\left(\sigma_{x} k_{y}-\sigma_{y} k_{x}\right)-\mu+\sigma_{y} h_{y} \Theta\left(\frac{L}{2}-|x|\right)$ and $\Delta(r)=\Delta_{0} e^{-i \frac{\varphi}{2}} \Theta\left(-x-\frac{L}{2}\right)+\Delta_{0} e^{i \frac{\varphi}{2}} \Theta\left(x-\frac{L}{2}\right)$, and $\Theta$ is the Heaviside step function, $\varphi$ is the random phase difference between two surface $S$ regimes. The details of the analytic calculation of Eq. (11) are shown in Ref. [40]. Here, we only discuss the main results. For both $\mu \rightarrow 0$ and $\mu \gg \Delta_{0}$, two branches of bound states have the same dispersion,

$$
\varepsilon\left(k_{y}\right)= \pm \sqrt{v_{F}^{2} k_{y}^{2}+\Delta_{0}^{2} \cos ^{2}\left(\frac{\varphi}{2}-\frac{h_{y} L}{v_{F}}\right)} .
$$

Here, $k_{y} \rightarrow 0$ for $\mu \rightarrow 0$, and $k_{y}=\mu / v_{F} \sin \theta$ with $\theta$ the incident angle approaching 0 . In equilibrium state with $\varphi=0$, a couple of gapless bound states can be obtained when $\frac{h_{y} L}{v_{F}}=\frac{\Delta \phi}{2}=\frac{(2 n+1) \pi}{2}$ with $n$ the integer number. Namely, 1D dispersive Majorana modes with constant density of states (DOS) are realized for the FM DWs with appropriate width $L$ and magnetization $h_{y}$. For finite $\mu$, the effective low-energy Hamiltonian $H_{\text {eff }}$ describing a couple of bound states can be obtained by projecting $H_{B d G}$ into the subspace spanned by two eigen-states $\xi_{ \pm}$ corresponding to $\varepsilon\left(k_{y}\right)=0$ with $k_{y}=0, \varphi=0, \frac{h_{y} L}{v_{F}}=$ $\frac{\pi}{2}[7]$, and $\xi_{ \pm}=\frac{1}{2}(\mp i,-1, \pm i, 1)^{T} e^{ \pm i \mu x / v_{F}-\int_{0}^{|x|} d \tilde{x} \Delta_{0}(\tilde{x}) / v_{F}}$. Then,

$$
H_{e f f}=\tilde{v}_{F} q_{y} \tau_{y}-\Delta_{0} \cos (\Delta \phi / 2) \tau_{z} .
$$

Here, $\tilde{v}_{F}=v_{F}\left[\cos k_{F} L+\left(\Delta_{0} / \mu\right) \sin k_{F} L\right] \Delta_{0}^{2} /\left(\mu^{2}+\Delta_{0}^{2}\right)$ with $\tau_{y}$ and $\tau_{z}$ the Pauli matrices spanned by particlehole space. When $\Delta \phi=\pi, H_{\text {eff }}$ in Eq. (44) still gives the 1D dispersive Majorana modes with constant DOS.

The implication for the experimental observations 36 . can be elucidated as follows. The calculated effective Zeeman energy $h_{y} \sim 55 \mathrm{meV}$. The width of DW is about twice about the lattice constant $L \sim 6.2 \AA$. The Fermi velocity $v_{F} \sim 216 \mathrm{meV} \AA[58]$. Thus the phase shift can be derived as $\Delta \phi \sim \pi$, which indicates that the 1D dispersive Majorana modes in $\mathrm{Fe}(\mathrm{Te}, \mathrm{Se})$ is likely to be induced due to the ferromagnetism of DWs. However, the realistic sample could not meet such rigorous condition of $\Delta \phi=\pi$. There should exist some slight fluctuations of $h_{y}$ and $L$ in the sample. Accordingly, $\Delta \phi$ should have tiny fluctuations around $\pi$. As a result, the experiment indeed observed the $d I / d V$ spectra showed some subtle differences at the different positions of DWs 36]. Such behavior is captured by the calculation [40], as shown in Fig. 2 (c). Besides, the picture of such surface S/F/S junction proposed here can also be verified by measure the tunneling current, which can be expressed as,

$$
I(\varphi, \Delta \phi)=-\frac{e N}{\hbar} \int_{-\pi / 2}^{\pi / 2} \frac{\partial\left|\varepsilon\left(k_{y}\right)\right|}{\partial \varphi} \tanh \frac{\left|\varepsilon\left(k_{y}\right)\right|}{2 k_{B} T} \cos \theta d \theta .
$$

Here, $k_{B}$ is Boltzmann constant. $N=k_{F} W / \pi$ denoting the number of the transport channels in the system with $W$ the length of the domain wall. As shown in Fig. 22(d), the tunneling current $I(\varphi, \Delta \phi)$ is none zero for $\varphi=0$ and $\Delta \phi \neq 0$ and $\pi$. Thus, the surface states of $\mathrm{FeSe}_{0.45} \mathrm{Te}_{0.55}$ with domain wall form a $\Delta \phi$ phase battery [59, 60].

According to Eq. (44), the boundary of the inverse masses can trap a MZM[7]. Thus, the surface S/F/S 

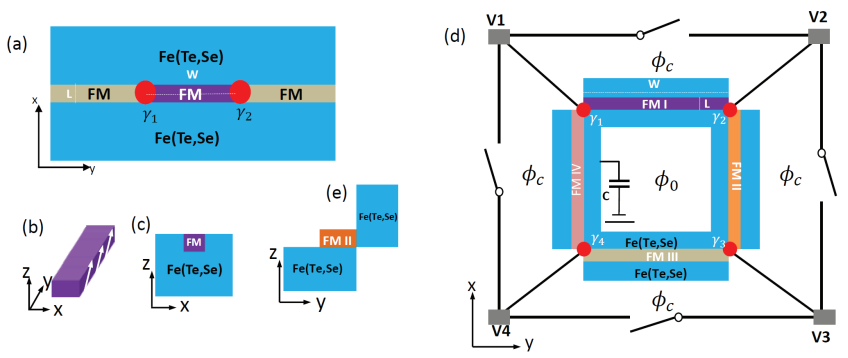

FIG. 3: (a) A basic device to create and fuse a pair of MZMs through tuning the magnetization of different regimes of the FM film. (b) The magnetic moments (white arrows) of the FM film can be tuned in the $y$-z plane by some external field. (c) The cross section of the middle part in (a). (d) The extended device built from the basic device. Four FM films I, II, III, IV are labeled. $\phi_{0}$ denotes the phase difference between the inner superconductor loop and the four outer superconductors. The superconductor is connected to ground by a capacitor. (e) The cross section of left edge of the square in (d).

junction can be upgraded by replacing the DWs with a thin FM film, as shown in Fig. 3 (a). According to $\Delta \phi=2 h_{y} L / v_{F}$, there are two ways to realize MZMs. The first one is to tune $h_{y}$ of different regimes of $\mathrm{F}$. In such case, the FM film can be fabricated with the soft magnetic materials, whose magnetization can be easily tuned by the electric, magnetic field etc., as shown in Fig. 3 (b). Note that the $z$-direction magnetization energy smaller than chemical potential and superconducting gap does not change the results. The second one is to tune $L$ of different regimes of $\mathrm{F}$, where the hard magnetic materials can be adopted to fabricate the FM film due to the robust magnetization. In both cases, the candidate magnetic materials could be selected among the yttrium iron garnet (YIG) ferrite, which includes many soft and hard ferrites such as $(\mathrm{MnZn}) \mathrm{O} \cdot \mathrm{Fe}_{2} \mathrm{O}_{3}$ and $\mathrm{BaO} \cdot 6 \mathrm{Fe}_{2} \mathrm{O}_{3}$, and the relevant film technique is very mature. Here, we adopt the first way, and the basic device is shown in Fig. 3 (a) and (c). Then, the mass term $m\left(h_{y}\right)=\Delta_{0} \cos \left(h_{y} L / v_{F}\right)$. Through tuning $h_{y}$ to have $m\left(h_{y}\right)<0$ in the middle regime of $\mathrm{F}$ and $m\left(h_{y}\right)>0$ in two side regimes, a pair of MZMs can be created at the boundaries between the middle and side regimes. They can also fuse by adiabatically tuning $m\left(h_{y}\right)$ to have same sign in all regimes. Namely, such basic device can realize the creation and fusion of a pair of MZMs through selectively tuning the magnetization $h_{y}$ of $\mathrm{F}$.

To realize and detect the braiding of the MZMs 61 69], the basic device has to involving two pairs of MZMs at least. The new device is shown in Fig. 3 (d) with the configuration of the cross section in Fig. 3 (e). Then, the superconducting square in Fig. 3 (e) disconnects with the outer superconductors and the phase difference is denoted by $\phi_{0}$. First, tuning $h_{y}$ to have $m\left(h_{y}\right)<0$ in I,
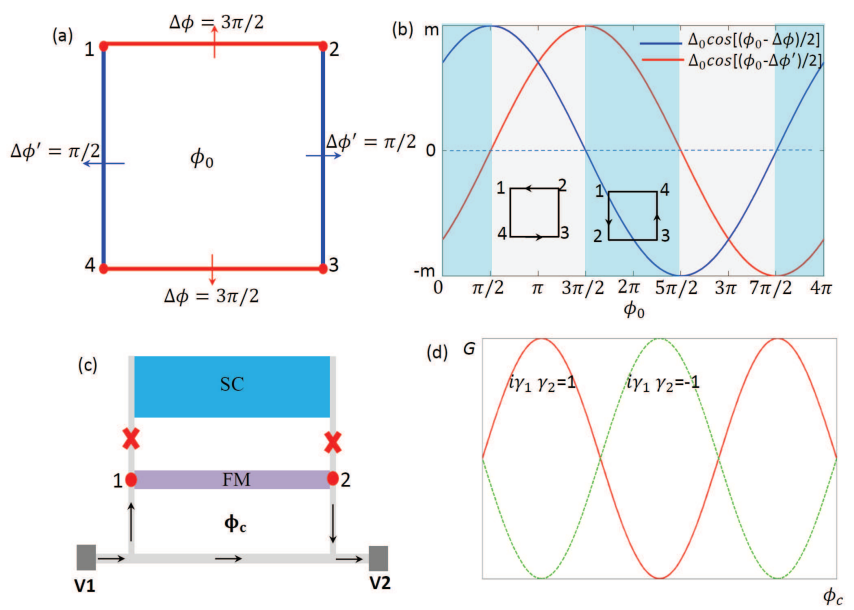

FIG. 4: (a) The square denotes the loop formed by the four FM films. $\Delta \phi=3 \pi / 2$ and $\Delta \phi^{\prime}=\pi / 2$ denote the values of $2 h_{y} L / v_{F}$ in regime I, III and II, IV, respectively. (b) The evolution of two masses $m^{(\prime)}=\Delta_{0} \cos \left[\left(\phi_{0}-\Delta \phi^{(\prime)}\right) / 2\right]$ as a phase difference $\phi_{0}$. The insert labels the equivalent exchange process of MZMs during the braiding. (c) The equivalent interference circuit to readout fermion parity of $i \gamma_{1} \gamma_{2}$. Note that the superconductor path is disconnected by the capacitor. (d) The interference oscillation of conductance as change as $\phi_{c}$ for different fermion parity.

III regimes and $m\left(h_{y}\right)>0$ in II, IV regimes as shown by the configuration in Fig. 4 (a), two pairs of MZMs labeled by $\gamma_{1}, \gamma_{2}, \gamma_{3}, \gamma_{4}$ are created and located on the four corners of the square. Two fermions can be defined as $f_{1 / 2}=\left(\gamma_{1 / 3}+i \gamma_{2 / 4}\right) / 2$. Suppose the initial state is occupied state of the two fermions, i.e., $|11\rangle=f_{1}^{\dagger} f_{2}^{\dagger}|00\rangle$ with $|00\rangle$ the vacuum state of both fermions. Second, the braiding operation is realized by adiabatically advancing the phase difference $\phi_{0}$ from 0 to $2 \pi$. During the process, the mass term $m=\Delta_{0} \cos \left[\left(\phi_{0}-\Delta \phi\right) / 2\right]$ also adiabatically evolves, as shown in Fig. 团 (b). This braiding operation is equivalent to exchange $\gamma_{2}$ and $\gamma_{4}$, i.e., $\gamma_{2} \rightarrow \gamma_{4}, \gamma_{4} \rightarrow$ $-\gamma_{2}$, as shown in the insert of Fig. 4 (b). Here, we assume the cut line is the right edge of the square [7]. The relevant braiding operator is $\mathcal{O}_{42}=\left(1+\gamma_{4} \gamma_{2}\right) / \sqrt{2}$, under which, the final state $\left|\Psi_{f}\right\rangle_{1}=\mathcal{O}_{42}|11\rangle=(|11\rangle-|00\rangle) / \sqrt{2}$. The twice braidings give $\left|\Psi_{f}\right\rangle_{2}=\mathcal{O}_{42}^{2}|11\rangle=-|00\rangle$, which means the fermion occupied number changes two. Note that the braiding result is unchanged by exchanging $\gamma_{1}$ and $\gamma_{3}$ and pinning $\gamma_{2}$ and $\gamma_{4}$. Third, the braiding results can be readout by electron teleportation effect of MZMs 61, 62] with the interference circuit in Fig. 3 (d). Take a pair of $\gamma_{1}, \gamma_{2}$ as an example. To detect the braiding results of fermion parity of $i \gamma_{1} \gamma_{2}$, one can connect the switch between $\gamma_{1}$ and $\gamma_{2}$ in Fig. 3 (d) and apply a magnetic flux $\phi_{c}$. The equivalent circuit is shown in Fig. 4 (c). The current flows along two different paths indicated by the arrows in Fig. 4 (c). Note that the super- 
conductor path can be forbidden by tuning the capacitor in Fig. 3 (d). Accordingly, the measured conductance should form interference oscillation as change as $\phi_{c}$, i.e., $G=g_{0}+i \gamma_{1} \gamma_{2} g_{1} \cos \left[e\left(\phi_{c}-\phi_{i}\right) / \hbar\right]$ with $\phi_{i}$ the intrinsic phase difference 62], and the fermion parity of $i \gamma_{1} \gamma_{2}$ can be readout, as shown in Fig. 4 (d) [61, 62]. The twice braiding can give the result of definitive sign change of $i \gamma_{1} \gamma_{2}$ between the initial and final states.

At last, we discuss the experimental feasibility and advantage of the designed device. First, the decay length of MZM is $\xi_{0} \sim \tilde{v}_{F} / \Delta_{0} \sim 7 \AA$ for $\mu=5 \Delta_{0}, v_{F} \sim 216$

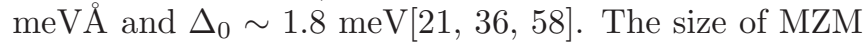
is very local. Second, the other quasi-particle energy is roughly estimated by $\sim \tilde{v}_{F} \pi / W[7]$. If the temperature $k_{B} T \sim 0.1 \mathrm{meV}$. The length of $W$ can be $\sim 27 \mathrm{~nm}$ for $\mu=5 \Delta_{0}$ and much larger than the decay length $\xi_{0}$. When temperature is below $1 \mathrm{~K} \sim 0.1 \mathrm{meV}$, the MZMs and relevant braiding are robust against the thermal fluctuation. The charging energy $U \sim Q_{0}^{2} / C$ is required to comparable to $\Delta_{0} \sim 1.8 \mathrm{meV}[61,62]$, which can lower the difficulty in control of the capacitor.

In conclusion, we propose a surface $\mathrm{S} / \mathrm{F} / \mathrm{S}$ junction to reveal the underlying physics of experimentally observed dispersive 1D Majorana mode in $\mathrm{FeSe}_{0.45} \mathrm{Te}_{0.55}$. Our calculations predict the spontaneously ferromagnetic order in the domain wall, and we prove the crucial role of ferromagnetism to modulate the superconducting phase of the surface electrons to form Majorana modes. More meaningfully, we design a feasible artificial device involving $\mathrm{FeSe}_{0.45} \mathrm{Te}_{0.55} /$ ferromagnet/ $\mathrm{FeSe}_{0.45} \mathrm{Te}_{0.55}$ junctions, which can create, fuse, braid and readout the Majorana zero modes.

We thank J. P. Hu, X. X. Wu, S. B. Zhang, S. S. Qin, F. W. Zheng, H. F. Du, L. Shan, Z. Y. Wang, S. C. Yan and X. Y. Hou for helpful discussions. This work was financially supported by the National Key R\&D Program of China No. 2017YFA0303201, National Natural Science Foundation of China under Grants (No. 12022413, No. 11674331, No.11625415), the "Strategic Priority Research Program (B)" of the Chinese Academy of Sciences, Grant No. XDB33030100, the '100 Talents Project' of the Chinese Academy of Sciences, the Collaborative Innovation Program of Hefei Science Center, CAS (Grants No. 2020HSC-CIP002), the CASHIPS Director's Fund (BJPY2019B03), the Science Challenge Project under Grant No. TZ2016001, the Major Basic Program of Natural Science Foundation of Shandong Province (Grant No. ZR2021ZD01). A portion of this work was supported by the High Magnetic Field Laboratory of Anhui Province, China.

* Electronic address: zhang ping@iapcm.ac.cn

$\dagger$ Electronic address: haon@hmfl.ac.cn
[1] D. A. Ivanov, Non-Abelian Statistics of Half-Quantum Vortices in p-Wave Superconductors. Phys. Rev. Lett. 86, 268 (2001).

[2] A. Y. Kitaev, Fault-tolerant quantum computation by anyons. Ann. Phys. 303, 2 (2003).

[3] A. Kitaev, Anyons in an exactly solved model and beyond. Ann. Phys. 321, 2 (2006).

[4] C. Nayak, S. H. Simon, A. Stern, M. Freedman, and S. Das Sarma, Non-Abelian anyons and topological quantum computation. Rev. Mod. Phys. 80, 1083 (2008).

[5] J. Alicea, New directions in the pursuit of Majorana fermions in solid state systems. Rep. Prog. Phys. 75, 076501 (2012).

[6] S. R. Elliott and M. Franz, Colloquium: Majorana fermions in nuclear, particle, and solid-state physics. Rev. Mod. Phys. 87, 137 (2015).

[7] L. Fu and C. L. Kane, Superconducting Proximity Effect and Majorana Fermions at the Surface of a Topological Insulator. Phys. Rev. Lett. 100, 096407 (2008).

[8] J. D. Sau, R. M. Lutchyn, S. Tewari, and S. Das Sarma, Generic New Platform for Topological Quantum Computation Using Semiconductor Heterostructures. Phys. Rev. Lett. 104, 040502 (2010).

[9] R. M. Lutchyn, J. D. Sau, and S. Das Sarma, Majorana Fermions and a Topological Phase Transition in Semiconductor-Superconductor Heterostructures. Phys. Rev. Lett. 105, 077001 (2010).

[10] Y. Oreg, G. Refael, and F. von Oppen, Helical Liquids and Majorana Bound States in Quantum Wires. Phys. Rev. Lett. 105, 177002 (2010).

[11] S. Nadj-Perge, I. K. Drozdov, J. Li, H. Chen, S. Jeon, J. Seo, A. H. MacDonald, B. A. Bernevig, and A. Yazdani, Observation of Majorana fermions in ferromagnetic atomic chains on a superconductor. Science 346, 602 (2014).

[12] N. Hao and J. Hu, Topological Phases in the Single-Layer FeSe. Phys. Rev. X 4, 031053 (2014).

[13] Z.Wang, P. Zhang, G. Xu, L. K. Zeng, H. Miao, X. Xu, T. Qian, H. Weng, P. Richard, A. V. Fedorov, H. Ding, X. Dai, and Z. Fang, Topological nature of the FeSe0.5Te0.5 superconductor. Phys. Rev. B 92, 115119 (2015).

[14] X. Wu, S. Qin, Y. Liang, H. Fan, and J. Hu, Topological characters in $\mathrm{Fe}\left(\mathrm{Te}_{1-x} \mathrm{Se}_{x}\right)$ thin films. Phys. Rev. B 93, 115129 (2016).

[15] N. Hao and J. Hu, Topological quantum states of matter in iron-based superconductors: from concept to material realization. Natl. Sci. Rev. 6, 213 (2019).

[16] X. L. Peng, Y. Li, X. X. Wu, H. B. Deng, X. Shi, W. H. Fan, M. Li, Y. B. Huang, T. Qian, P. Richard, J. P. Hu, S. H. Pan, H. Q. Mao, Y. J. Sun, and H. Ding, Observation of topological transition in high-Tc superconducting monolayer $\mathrm{FeTe}_{1-x} \mathrm{Se}_{x}$ films on $\mathrm{SrTiO}_{3}(001)$. Phys. Rev. B 100, 155134 (2019).

[17] P. Zhang, Z. Wang, X. Wu, K. Yaji, Y. Ishida, Y. Kohama, G. Dai, Y. Sun, C. Bareille, K. Kuroda, T. Kondo, K. Okazaki, K. Kindo, X. Wang, C. Jin, J. Hu, R. Thomale, K. Sumida, S.Wu, K. Miyamoto, T. Okuda, H. Ding, G. D. Gu, T. Tamegai, T. Kawakami, M. Sato, and S. Shin, Multiple topological states in iron-based superconductors. Nat. Phys. 15, 41 (2019).

[18] X. Wu, S. Qin, Y. Liang, C. Le, H. Fan, and J. Hu, $\mathrm{CaFeAs}_{2}$ : A staggered intercalation of quantum spin Hall and high-temperature superconductivity. Phys. Rev. B 91, 081111(R) (2015). 
[19] G. Xu, B. Lian, P. Tang, X.-L. Qi, and S.-C. Zhang, Topological Superconductivity on the Surface of FeBased Superconductors. Phys. Rev. Lett. 117, 047001 (2016).

[20] X. Shi, Z.-Q. Han, P. Richard, X.-X. Wu, X.-L. Peng, T. Qian, S.-C.Wang, J.-P. Hu, Y.-J. Sun, and H. Ding, Enhanced superconductivity accompanying a Lifshitz transition in electron-doped FeSe monolayer. Sci. Bull. 62, 503 (2017).

[21] P. Zhang, K. Yaji, T. Hashimoto, Y. Ota, T. Kondo, K. Okazaki, Z.Wang, J.Wen, G. D. Gu, H. Ding, and S. Shin, Observation of topological superconductivity on the surface of an iron-based superconductor. Science $\mathbf{3 6 0}$, 182 (2018).

[22] Q. Wang, C.-C. Liu, Y.-M. Lu, and F. Zhang, HighTemperature Majorana Corner States. Phys. Rev. Lett. 121, 186801 (2018).

[23] Z. Yan, F. Song, and Z. Wang, Non-Hermitian Chern Bands. Phys. Rev. Lett. 121, 096803 (2018).

[24] R.-X. Zhang, W. S. Cole, and S. Das Sarma, Helical Hinge Majorana Modes in Iron-Based Superconductors. Phys. Rev. Lett. 122, 187001 (2019).

[25] X. Wu, X. Liu, R. Thomale, and C.-X. Liu, High-Tc Superconductor $\mathrm{Fe}(\mathrm{Se}, \mathrm{Te})$ Monolayer: an Intrinsic, Scalable and Electrically-tunable Majorana Platform. Natl. Sci. Rev. nwab087 (2021).

[26] R.-X. Zhang,W. S. Cole, X.Wu, and S. Das Sarma, Higher-Order Topology and Nodal Topological Superconductivity in $\mathrm{Fe}(\mathrm{Se}, \mathrm{Te})$ Heterostructures. Phys. Rev. Lett. 123, 167001 (2019).

[27] J. X. Yin, Z. Wu, J. H. Wang, Z. Y. Ye, J. Gong, X. Y. Hou, L. Shan, A. Li, X. J. Liang, X. X. Wu, J. Li, C. S. Ting, Z. Q. Wang, J. P. Hu, P. H. Hor, H. Ding, and S. H. Pan, Observation of a robust zero-energy bound state in iron-based superconductor $\mathrm{Fe}(\mathrm{Te}, \mathrm{Se})$. Nat. Phys. 11, 543 (2015).

[28] D. Wang, L. Kong, P. Fan, H. Chen, S. Zhu, W. Liu, L. Cao, Y. Sun, S. Du, J. Schneeloch, R. Zhong, G. Gu, L. Fu, H. Ding, and H.-J. Gao, Evidence for Majorana bound states in an iron-based superconductor. Science 362, 333 (2018).

[29] Q. Liu, C. Chen, T. Zhang, R. Peng, Y.-J. Yan, C.-H.P. Wen, X. Lou, Y.-L. Huang, J.-P. Tian, X.-L. Dong, G.-W. Wang, W.-C. Bao, Q.-H. Wang, Z.-P. Yin, Z.-X. Zhao, and D.-L. Feng, Robust and Clean Majorana Zero Mode in the Vortex Core of High-Temperature Superconductor $\left(\mathrm{Li}_{0.84} \mathrm{Fe}_{0.16}\right)$ OHFeSe. Phys. Rev. X 8, 041056 (2018).

[30] T. Machida, Y. Sun, S. Pyon, S. Takeda, Y. Kohsaka, T. Hanaguri, T. Sasagawa, and T. Tamegai, Zero-energy vortex bound state in the superconducting topological surface state of Fe(Se,Te). Nat. Mater. 18, 811 (2019).

[31] W. Liu, L. Cao, S. Zhu, L. Kong, G. Wang, M. Papaj, P. Zhang, Y.-B. Liu, H. Chen, G. Li, F. Yang, T. Kondo, S. Du, G.-H. Cao, S. Shin, L. Fu, Z. Yin, H.-J. Gao and H. Ding, A new Majorana platform in an Fe-As bilayer superconductor. Nat. Commun. 11, 5688 (2020).

[32] C. Liu, C. Chen, X. Liu, Z. Wang, Y. Liu, S. Ye, Z. Wang, J. Hu and J. Wang, Zero-energy bound states in the high-temperature superconductors at the twodimensional limit. Sci. Adv. 6, eaax7574 (2020).

[33] C. Chen K. Jiang, Y. Zhang, C. Liu, Y. Liu, Z. Wang and J. Wang, Atomic line defects and zero-energy end states in monolayer $\mathrm{Fe}(\mathrm{Te}, \mathrm{Se})$ high-temperature superconduc- tors Nat. Phys. 16, 536 (2020).

[34] X. Wu, J.-X. Yin, C.-X. Liu, and J. Hu, Topological magnetic line defects in $\mathrm{Fe}(\mathrm{Te}, \mathrm{Se})$ high-temperature superconductors, arXiv:2004.05848

[35] Y. Zhang, K. Jiang, F. Zhang, J. Wang, and Z. Wang, Atomic Line Defects and Topological Superconductivity in Unconventional Superconductors. Phys. Rev. X 11, 011041 (2021).

[36] Z. Wang, J. O. Rodriguez, L. Jiao, S. Howard, M. Graham, G. D. Gu, T. L. Hughes, D. K. Morr, V. Madhavan, Evidence for dispersing 1D Majorana channels in an ironbased superconductor. Science 367, 104 (2020).

[37] P. Fulde and R. A. Ferrell, Superconductivity in a Strong Spin-Exchange Field. Phys. Rev. 135, A550 (1964).

[38] A. I. Larkin, and Y. N. Ovchinnikov, Nonuniform state of superconductors. Zh. Eksp. Teor. Fiz. 47, 1136-1146 (1964).

[39] Private communication.

[40] See Supplemental Material at http://xxxx for details about the first principles calculating methods, derivation of Adereev bound state, Majornan zero-energy-mode model, and the STS spectrum (see also Refs. [41-50] therein).

[41] G. Kresse and J. Furthmüller, Efficient iterative schemes for ab initio total-energy calculations using a plane-wave basis set. Phys. Rev. B 54, 11169 (1996).

[42] G. Kresse and D. Joubert, From ultrasoft pseudopotentials to the projector augmented-wave method. Phys. Rev. B 59, 1758 (1999).

[43] P. E. Blchl, Projector augmented-wave method. Phys. Rev. B 50, 17953 (1994).

[44] J. P. Perdew, K. Burke, and M. Ernzerhof, Generalized Gradient Approximation Made Simple. Phys. Rev. Lett. 77, 3865 (1996).

[45] Jacob Linder, Yukio Tanaka, Takehito Yokoyama, Asle Sudbo, and Naoto Nagaosa, Unconventional superconductivity on a topological insulator. Phys. Rev. B 81, 184525 (2010).

[46] A. Buzdin, L. N. Bulaevskii, S. Panyukov, Criticalcurrent oscillations as a function of the exchange field and thickness of the ferromagnetic metal (F) in an SF-S Josephson junction. Pis'Ma Zh. Eksp. Teor. Fiz 35. 147-148 (1982).

[47] A. I. Buzdin, Proximity effects in superconductorferromagnet heterostructures. Rev. Mod. Phys. 77, 935 (2005).

[48] R. C. Dynes, J. P. Garno, G. B. Hertel, and T. P. Orlando, Tunneling Study of Superconductivity near the Metal-Insulator Transition. Phys. Rev. Lett 53, 2437 (1984)

[49] Q. Gu, Y. Li, S. Wan, H. Li, W. Guo, H. Yang, Q. Li, X. Zhu, X. Pan, Y. Nie and H.-H. Wen, Single particle tunneling spectrum of superconducting $\mathrm{Nd}_{1-x} \mathrm{Sr}_{x} \mathrm{NiO}_{2}$ thin films Nat Commun 11, 6027 (2020)

[50] W. Duan, K. Chen, W. Hong, X. Chen, H. Yang, S. Li, H. Luo, and H.-H. Wen, Single-particle tunneling spectroscopy and superconducting gaps in the layered ironbased superconductor $\mathrm{KCa}_{2} \mathrm{Fe}_{4} \mathrm{As}_{4} \mathrm{~F}_{2}$. Phys. Rev. B 103, 214518 (2021)

[51] F. Ma, W. Ji, J. Hu, Z.-Y. Lu, and T. Xiang, FirstPrinciples Calculations of the Electronic Structure of Tetragonal $\alpha$-FeTe and $\alpha$-FeSe Crystals: Evidence for a Bicollinear Antiferromagnetic Order. Phys. Rev. Lett. 102, 177003 (2009). 
[52] K. Liu, Z.-Y. Lu, and T. Xiang, Nematic antiferromagnetic states in bulk FeSe. Phys. Rev. B 93, 205154 (2016).

[53] J. K. Glasbrenner, I. I. Mazin, Harald O. Jeschke, P. J. Hirschfeld, Roser Valentí, Effect of magnetic frustration on nematicity and superconductivity in iron chalcogenides. Nat. Phys. 11, 953 (2015).

[54] P. Chandra, P. Coleman and A. I. Larkin, Ising transition in frustrated Heisenberg models. Phys. Rev. Lett. 64, 88 (1990).

[55] J. Hu, B. Xu, W. Liu, N. Hao, Y. Wang, Unified minimum effective model of magnetic properties of iron-based superconductors. Phys. Rev. B 85, 144403 (2012).

[56] N. J. McLaughlin, H. Wang, M. Huang, E. Lee-Wong, L. Hu, H. Lu, G. Q. Yan, G. Gu, C. Wu, Y.-Z. You, and C. R. Du, Strong Correlation Between Superconductivity and Ferromagnetism in an Fe-Chalcogenide Superconductor. Nano Lett. 21, 7277 (2021).

[57] N. Zaki, G. Gu, A. Tsvelik, C. Wu, and P. D. Johnson, PNAS 118, e2102165118 (2021).

[58] D. Wang, L. Kong, P. Fan, H. Chen, S. Zhu, W. Liu, L. Cao, Y. Sun, S. Du, J. Schneeloch, R. Zhong, G. Gu, L. Fu, H. Ding, H.-J. Gao, Evidence for Majorana bound states in an iron-based superconductor. Science 362, 333 (2018).

[59] A. Buzdin, Direct coupling between magnetism and superconducting current in the Josephson $\varphi_{0}$ junction. Phys. Rev. Lett. 101, 107005 (2008).

[60] E. Strambini, A. Iorio, O. Durante, R. Citro, C. SanzFernández, C. Guarcello, I. V. Tokatly, A. Braggio, M. Rocci, N. Ligato, V. Zannier, L. Sorba, F. S. Bergeret, and F. Giazotto, A Josephson phase battery. Nat. Nanotechnol. 15, 656 (2020).
[61] L. Fu, Electron teleportation via Majorana bound states in a mesoscopic superconductor. Phys. Rev. Lett. 104, 056402 (2010).

[62] S. Vijay and L. Fu, Teleportation-based quantum information processing with Majorana zero modes. Phys. Rev. B 94, 235446 (2016).

[63] J. Alicea, Y. Oreg, G. Refael, F. von Oppen, M. P. A. Fisher, Unconventional Josephson signatures of Majorana bound states. Nat. Phys. 7412 (2011).

[64] P. Bonderson, M. Freedman, C. Nayak, Measurementonly topological quantum computation. Phys. Rev. Lett. 101010501 (2008).

[65] T. Karzig, C. Knapp, R. M. Lutchyn, P. Bonderson, M. B. Hastings, C. Nayak, J. Alicea, K. Flensberg, S. Plugge, Y. Oreg, C. M. Marcus, and M. H. Freedman, Scalable designs for quasiparticle-poisoning-protected topological quantum computation with Majorana zero modes. Phy. Rev. B 95, 235305 (2017).

[66] R. AguadoLa, Majorana quasiparticles in condensed matter. Rivista del Nuovo Cimento 40, 523 (2017).

[67] R. AguadoLa, and L. P. Kouwenhoven, Majorana qubits for topological quantum computing. Physics Today 73, $44(2020)$.

[68] S.-B. Zhang, A. Calzona, and B. Trauzettel, Allelectrically tunable networks of Majorana bound states. Phys. Rev. B 102, 100503(R) (2020).

[69] S.-B. Zhang, W. B. Rui, A. Calzona, S.-J. Choi, A. P. Schnyder, and B. Trauzettel, Topological and holonomic quantum computation based on second-order topological superconductors. Phys. Rev. Research 2, 043025 (2020). 